Educa-UMCH,15, enero - junio 2020 https://doi.org/ 10.35756/educaumch.202015.139 ISSN: 2617-0337

\title{
Tecnologías de la información y la comunicación como mediadoras del aprendizaje: desafíos actuales
}

\section{Information and communication technologies as} mediators of learning: current challenges

Recibido: 05 de enero 2020, Evaluado: 04 de febreo 2020, Aceptado: 03 de junio 2020

Rossana Fiorella Felipa Andrade felipaandrader@gmail.com https://orcid.org/0000-0001-6721-5928

Universidad Privada del Norte

\section{Como citar el artículo}

Felipa Andrade, R. (2020). Tecnologías de la información y la comunicación como mediadoras del aprendizaje: desafíos actuales. Revista EDUCA UMCH, (15), 160169. https://doi.org/10.35756/educaumch.202015.139

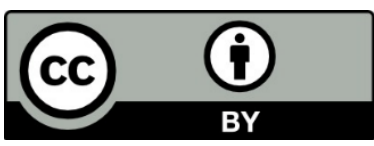

Esta obra está bajo una licencia internacional Creative Commons Atribución 4.0. 


\section{Resumen}

El aprendizaje y las diversas formas de aprender han sido siempre objeto de preocupacióny de investigación en el campo educativo. La presencia de las tecnologías de la información y la comunicación (TIC) y su inserción en los procesos de enseñanza- aprendizaje han derivado en la gestación de nuevas teorías de aprendizaje, como el Conectivismo, y, simultáneamente, en la generación de diversos desafíos, tanto para las instituciones educativas como para docentes y estudiantes. En este artículo, se intenta describir la relación entre la tecnología y la educación, a partir de la revisión de sus áreas de desarrollo y de la identificación de los principales retos que se afrontan actualmente en las aulas de clase.

Palabras claves: Aprendizaje, Conectivismo, desafios, TIC

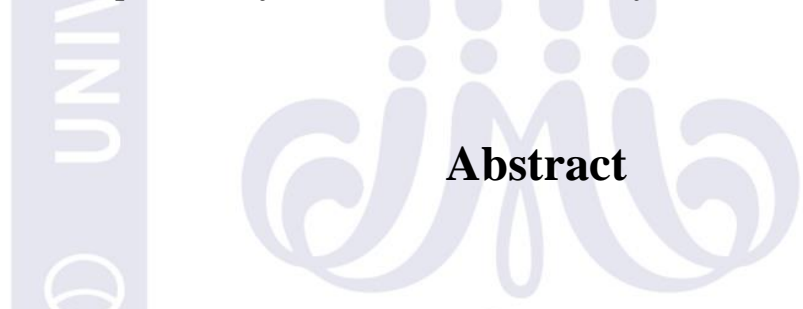

Learning and different ways of learning have always been the subjetc of concern and research in the educational field. The presence of information and communication technologies (ICT) and its insertion in the teaching-learning processes have resulted in the creation of new learning theories, such as Connectivism, and, simultaneously, the generation of various challenges, both for educational institutions and for teachers and students. In this article, we try to describe the relationship between technology and education, based on the review of their development areas and the identification of the main challenges currently facing classrooms.

Key words: Learning, Connectivism, challenges, ICT 


\section{Introducción}

En el marco del estudio de las Tecnologías de la información y la comunicación, y su implicancia en los procesos de enseñanza-aprendizaje, "las TIC se entienden como recursos que tienen un alto potencial para favorecer aprendizajes y para implementar propuestas innovadoras de enseñanza, más allá de que sus fines originales no hayan estado basados en supuestos pedagógicos" (International Institute for Educational Planning (IIEP), 2006, p. 33)

Precisamente, si bien hoy en día es muy común referirse a las TIC en el campo educativo, es sabido que su surgimiento no se dio en escenarios académicos. Sin embargo, de un tiempo hasta aquí, su presencia en contextos donde se desarrollan procesos de enseñanza- aprendizaje cobra cada vez más fuerza, aunque resulta necesario delimitar muy bien sus fines, las formas de incorporarlas y definir, así como justificar muy bien su intervenciónen procesos académicos sumamente relevantes que determinan la formación integral de los educandos de nuestro país.

La interrogante principal en este punto es la siguiente: ¿cuál es rol de la tecnología en la educación? A partir de este cuestionamiento, procederé a proponer una reflexión que tomecomo punto de partida la verdadera necesidad de la incorporación de las TIC en el escenario educativo $\mathrm{y}$, posteriormente, describiré algunos desafíos que implica su presencia en las aulas de clase, los cuales, a mi parecer, determinan su acción y los resultados de los procesos de enseñanza-aprendizaje.

\section{Principales dominios de la tecnología en el campo educativo}

La presencia de la tecnología en nuestras vidas es un hecho innegable. El individuo promedio del siglo XXI gestiona sus actividades (personales, académicas, laborales, etc.) con el soporte de dispositivos y aplicaciones inteligentes que le posibilitan organizar información de forma funcional y en tiempo real. Esta premisa no intenta generalizar tal condición a toda la humanidad, pues hoy en día, es posible hallar personas que viven apartados de la tecnología y no se sienten, necesariamente, en desventaja por ello.

Específicamente, en el campo de la educación, las instituciones educativas se encuentran concentradas en efectuar esfuerzos para incorporar/implementar/potenciar, según sea el caso, distintas herramientas tecnológicas conducentes a acciones educativas innovadoras, tan anheladas y apreciadas por las comunidades académicas. En ese camino, surge un primer punto de quiebre, de mención necesaria e inevitable: ¿Cuál es el rol que asume la tecnología en el marco de la formación de los futuros profesionales de nuestra sociedad? 
Linn (2002, p. 349) intenta responder esta interrogante al señalar que "usar la tecnología para crear entornos de aprendizaje y luego estudiar cómo los estudiantes, profesores e investigadores los aprovechan ofrece un camino prometedor para desarrollar usos mejores". La esencia, entonces, es generar escenarios de aprendizaje favorables para el desarrollo de competencias generales y específicas en las distintas áreas de conocimientoque conforman el currículo. La premisa resulta interesante, por lo que habría que evaluarcuál sería el papel de estas herramientas si estos escenarios no logran producir, finalmente, aprendizajes dirigidos a la formación integral que las instituciones desean alcanzar en la población estudiantil que atiende.

Por su parte, Meneses y Velasco (2007, p. 56) sostienen que este rol podría estar orientado a "renovar sustantivamente los procesos formativos en múltiples formas y alterar, en consecuencia, las formas de comunicación entre docentes y alumnado, así como los procesos de enseñanza y aprendizaje". Esta afirmación nos dirige a una reflexión importante, puntualmente, en lo que se refiere a la disposición de estos agentes protagonistas para incorporar prácticas tecnológicas en su rutina académica y a las competencias con las que cuentan para afrontar de forma óptima los cambios implicados en este proceso.Al respecto, considero que una primera acción estratégica recae directamente en la labor del profesor, por cuanto este debería contemplar una programación de actividades flexible y funcional, y estar preparado para responder de forma oportuna y apropiada a las posibles dudas por parte de los estudiantes, con el soporte de rutas abiertas de comunicación e intercambio de información (Meneses y Velasco, 2007, p. 57)

A partir de lo expuesto anteriormente, recojo el aporte de Czerwonogora (2011, p. 235), quien se apoya en la propuesta de Siemens para respaldar uno de los principios más importantes de la teoría conectivista: las personas "aprendemos haciendo conexiones conotras personas, conceptos e ideas". Efectivamente, las conexiones a las que se hace referencia pueden surgir en distintos escenarios y modalidades: en el plano social, "cara a cara" o a través de herramientas tecnológicas funcionales. Así, es posible revisar las áreas de desarrollo de la tecnología en la educación (Downes, como se citó en Czerwonogora, 2011, p. 236).

Los dominios de la tecnología en el escenario educativo son el conocimiento, el aprendizaje y la comunidad. En principio, el conocimiento puede describirse como la información concretada en un sinnúmero de conexiones entre los seres humanos, a partir de interacciones sociales y de las correlaciones entre los individuos y los artefactos/dispositivos con los que se comunican. Por su parte, el aprendizaje es entendido, en esta perspectiva, como la creación de conocimiento entre individuos o sociedades de manera dinámica y con valores adicionales como la retroalimentación y la armonía en la comunidad. Precisamente, el último elemento en este conjunto de dominios es la comunidad, cuyo fin es generar conexiones y aprendizajes exitosos, gracias a dos condiciones claves: 1). disposición para aprender de otros y con otros, y 2). mentalidad abierta para adaptarse a la dinámica cambiante de la información. 
Es claro que estos componentes no se desenvuelven de manera aislada, sino más bien, se complementan en la generación de conocimientos en redes interconectadas de aprendizaje. La siguiente figura representa esta relación:

Figura 1. Áreas de dominio de la tecnología en la educación

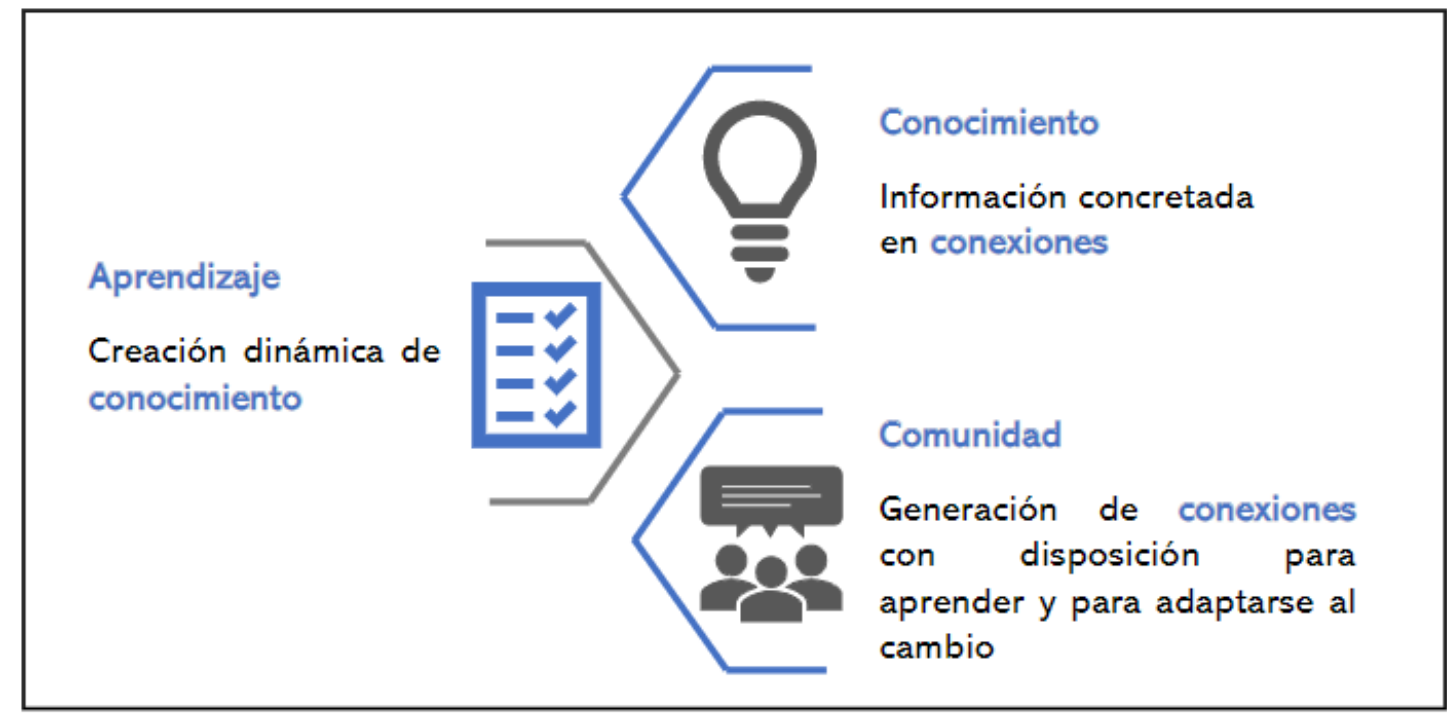

Elaboración propia a partir de lo expuesto sobre conocimiento, aprendizaje y comunidad (Czerwonogora, 2011)

\section{Conectivismo}

En el apartado anterior, se mencionó a Siemens y a la teoría que este investigador desarrolló: el conectivismo. Una primera premisa que intenta definir esta teoría es que "elaprendizaje ha dejado de ser una actividad interna e individual" (Siemens \& Leal, 2013,

p. 9). Si bien el aporte de cada individuo en su sociedad es importante, está claro que, actualmente, el conocimiento y la información es variable y que lo que puede ser útil hoy, en términos de información, mañana puede dejar de serlo. En esa línea, Stephen Downes,filósofo y comentarista sobre aprendizaje en nuevos medios de comunicación, señala lo siguiente: "(...) the term connectivism describes a form of knowledge and a pedagogy based on the idea that knowledge is distributed across a network of connections and that learning consists of the ability to construct and traverse those networks" (Downes, 2008).Efectivamente, lo que el autor señala, concretamente, es que el conocimiento se distribuye en redes de conexiones y que el aprendizaje es una habilidad de construcción con el soporte de dichas redes. Hasta este punto, Siemens (2013) y Downes (2008) coinciden en un principio trascendental: las formas individuales de aprender se ven relegadas frente a la posibilidad de adquirir nuevo conocimiento con la ayuda de redes de aprendizaje, especialmente en esta, la llamada era digital.

revistaeduca@umch.edu.pe

revistas.umch.edu.pe 
Por lo anterior, la concepción de aprendizajes en el plano individual ha perdido fuerza, por cuanto la acción de aprender se fortalece a partir de conexiones en comunidades. Estadinámica también se ve reforzada por la autonomía y la diversidad en las redes o comunidades de aprendizaje, en la medida que surge una valiosa interacción de perspectivas completamente diferentes sobre el conocimiento. Cada uno de estos aportescontribuyen en la construcción de conocimientos y fortalecen nuevas formas de aprendizaje.

La siguiente figura representa la interacción e integración de la comunidad de aprendizaje con los diversos recursos con los que cuenta: información, conocimiento, interacción.

Figura 2. Integración de una comunidad de aprendizaje

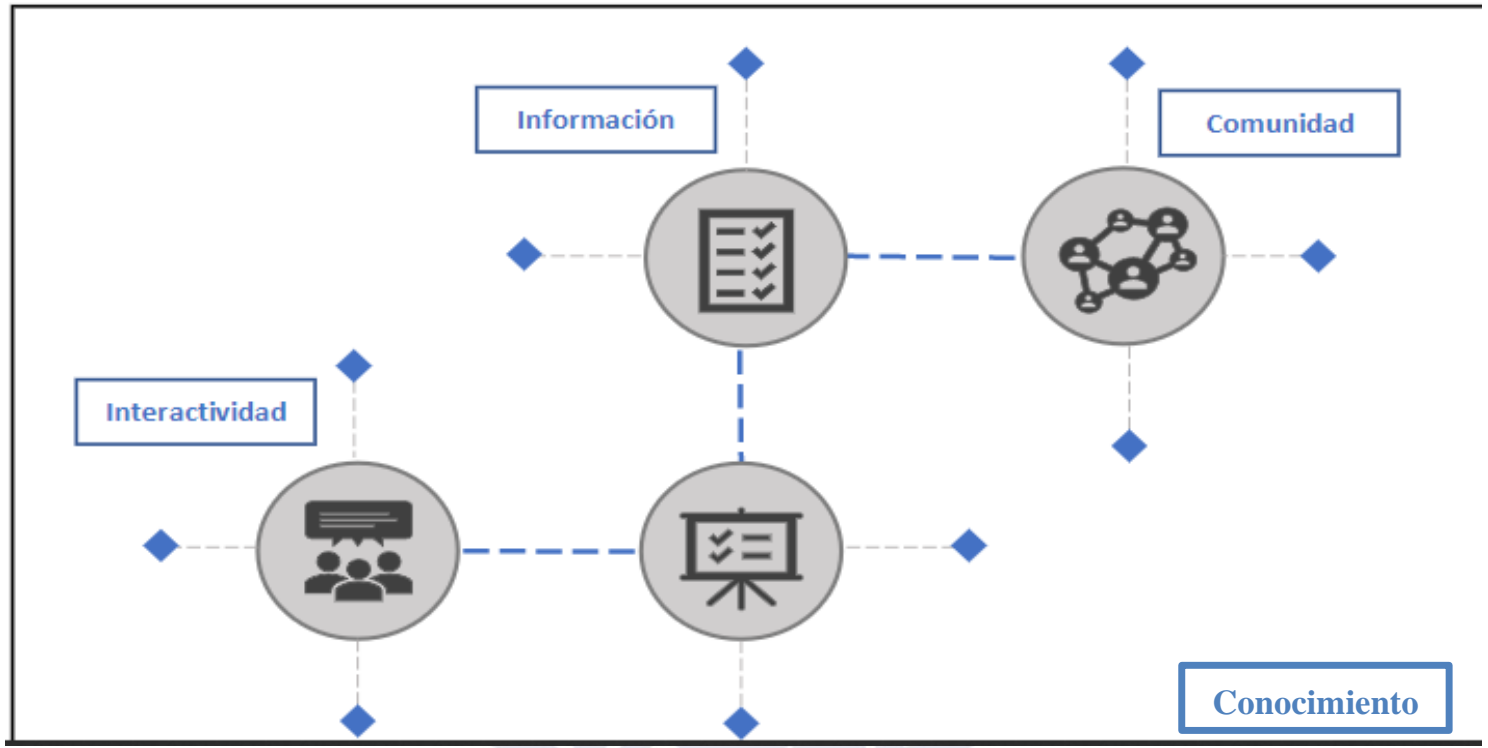

Elaboración propia a partir de lo expuesto por Downes (2008) y Siemens (2013)

Frente a las nuevas formas de aprender, las instituciones educativas y los agentes protagonistas cuentan con nuevas posibilidades de optimizar los procesos académicos deenseñanza-aprendizaje, pero también enfrentan algunos desafíos.

\section{Desafíos actuales}

\section{Necesidad versus esnobismo}

Usualmente, las TIC son consideradas como recursos motores del cambio y de la innovación educativa. Entonces, el primer paso para alcanzar resultados reales y funcionales en nuestra educación es dejar de pensar de esta forma. Para ello, es fundamental trascender al esnobismo y actuar según las demandas y las necesidades de nuestra sociedad. Con intención de clarificar el panorama, debe entenderse como 
esnobismo a la admiración desproporcionada por aquello que se considera una novedad o que "está de moda". De esta forma, el foco de atención debe ubicarse en el rol del docente y en su acción en el salón de clase: la selección de recursos tecnológicos debe pasar por las decisiones didácticas del docente y no por las bondades del recurso en sí mismo (International Institute for Educational Planning (IIEP), 2006, p. 33).

Las TIC son recursos para la enseñanza, pero el eje de las decisiones sobre cómo, por qué y cuándo incorporarlas responde más a decisiones didácticas que a decisiones vinculadascon sus características como recursos. En ese sentido, es conveniente que los maestros se encuentren convencidos sobre la funcionalidad y pertinencia de estos recursos y que se respalden en este conocimiento para la toma oportuna de decisiones académicas.

\section{Aprender, desaprender y reaprender}

Pese a lo anterior, en palabras de Coll (2009, p. 1) se presenta un “(...) desfase existente entre, por una parte, las elevadas expectativas de cambio y mejora de la educación escolar generadas por estas tecnologías, y por otra, los limitados avances conseguidos hasta el momento". Ello nos permite preguntarnos: ¿la presencia de las TIC son realmente la solución frente a la crisis educativa que afrontamos actualmente? Sin necesidad de realizar búsquedas incansables por hallar el meollo del asunto, considero que la solución somos nosotros: los educadores. Si bien no es una tarea fácil, es pertinente aprender, desaprender y reaprender. El incorporar las TIC en los entornos de enseñanza-aprendizaje implica un proceso complejo, en el que los principales agentes educativos, docentes y estudiantes, tienen que desligarse de los patrones que han regido sus actuaciones, serflexibles a los cambios y sentir una disposición genuina para dejar atrás los rolestradicionales asumidos (Cabero, 2005).

Entonces, y según lo expresado por los autores antes mencionados, el camino debe estar delimitado por un cambio profundo de mentalidad y por actuaciones que respondan a lasnecesidades reales de los protagonistas del acto educativo: maestros y estudiantes. Los alumnos se encuentran frente a un gran reto: desligarse completamente del rol pasivo asumido hasta el momento y desafiar las formas convencionales de aprender por otras formas en las que sean protagonistas reales de los procesos didácticos realizados en las aulas. Por su parte, el docente es consciente de que ha dejado definitivamente el papel detransmisor de contenidos para convertirse en un dinamizador y orientador de los procesosde enseñanza-aprendizaje.

Así, los actores principales del escenario educativo se encuentran en la dirección de un cambio de mentalidad necesario y crucial para que los objetivos estratégicos del ProyectoEducativo Nacional (PEN) se cumplan a cabalidad. 


\section{Aprendizaje en constante transformación}

La enseñanza y el aprendizaje en el marco de la presencia e incorporación de las TIC en el contexto educativo actual nos ubica frente a otro gran desafío: el aprendizaje se transforma permanentemente. En esta línea, la educación del alumnado debe estar dirigida a desarrollar personas “(...) no tanto con habilidad en la búsqueda de información, sino más para su selección, evaluación y adaptación a su problema educativo(...)" (Cabero, 2005). Lo anterior significa que el trabajo de fondo radica en orientar a los estudiantes a un uso consciente, discriminado y funcional de la información que tienen a su alcance, siempre en respuesta a las necesidades de su entorno. En consecuencia, es irrelevante contar con una cantidad infinita de contenidos diversos si no se ha formado estudiantes conscientes y discriminadores de los recursos que tienen al frente.

Para concluir, es importante reiterar que las tecnologías no han llegado para salvarnos. Tanto los estudiantes como los docentes somos responsables de que la presencia e intervención de las tecnologías de la información y la comunicación (TIC) conduzcan a concretar aprendizajes funcionales para la vida de las personas. La presencia de estos recursos no es la solución, en cambio, su correcta gestión y aprovechamiento oportuno pueden ser claves para concretar una educación de calidad, aquella tan anhelada en los tiempos actuales.

\section{Conclusiones}

El proceso de aprendizaje no es estático, más bien, cambia constantemente, especialmentesi se le relaciona con la incorporación de las tecnologías de información y la comunicación (TIC). El cuestionamiento principal en este artículo está dirigido a definircuál es el rol de la tecnología en los procesos académicos y qué desafíos deben enfrentarlos principales protagonistas de dichos procesos. Por un lado, las TIC poseen innegables características y condiciones que podrían favorecer sustancialmente el aprendizaje de los estudiantes; así lo establece la teoría conectivista, la cual postula, esencialmente, que el aprendizaje se genera por las conexiones surgidas en una comunidad de aprendizaje cara a cara o virtual, no obstante, su inserción debe responder a objetivos claros, a formas coherentes de incorporación y a una intervención pertinente y oportuna según las necesidades que presenta el alumnado. Por otra parte, los desafíos más grandes por enfrentar están relacionados con hallar las demandas urgentes y reales de la población estudiantil para arribar a una selección precisa, oportuna y responsable de aquellas herramientas que intervendrán en la construcción de los aprendizajes. Adicionalmente, es conveniente aprender de estos elementos tecnológicos y repensar en los roles que han venido desempeñando docentes y estudiantes. Finalmente, se encuentra una de lascondiciones más visibles en la tarea de aprender y enseñar: el aprendizaje y la información se encuentran en constante cambio, por lo que la formación en la búsqueda discriminaday funcional de contenidos se vuelve crucial en el escenario educativo. 


\section{Referencias}

Cabero, J. (2005). Las TIC y las universidades: retos, posibilidades y preocupaciones. Revista de La Educación Superior, 34, 77-100. https://doi.org/CurricularTICs.pdf

Coll, C. (2009). Aprender y enseñar con las TIC. En Carneiro, R., Toscano, J.C., Díaz, T. Los Desafíos de Las TIC Para El Cambio Educativo., 113-126.

Czerwonogora, A. (2011). El aprendizaje en la era digital. Revista Electrónica de Diálogos Educativos, 11(21), 3-20.

Downes, S. (2008). Places to Go: Connectivism \&amp; Connective Knowledge Recommended APA Citation. Innovate: Journal of Online Education, 5(1). Retrieved from https://nsuworks.nova.edu/innovate/vol5/iss 1/6

International Institute for Educational Planning (IIEP). (2006). La integración de las Tecnologías de la Información y la Comunicación en los Sistemas Educativos. Retrieved from http://unesdoc.unesco.org/images/0015/001507/150785s.pdf

Linn, M. (2002). . Promover la educación científica a través de las tecnologías de la información y comunicación (TIC). Enseñanza de Las Ciencias: Revista de Investigación y Experiencias Didácticas, 20(3), 347-356.

Meneses, E. L., \& Velasco, M. J. M. (2007). Rol del profesorado y en los procesos de enseñanza- the influence of the information technology on the role of teachers and on Eloy López Meneses María Jesús Miranda Velasco Tal como señala Barry Wellman ( 2004 ) 1 en TheInternet in Everyday Life : An. (1138-2783), 51-60. Retrieved from https://www.utpl.edu.ec/ried/images/pdfs/inlfuencia.pdf

Siemens, George; Leal Fonseca, D. E. (2013). Conectivismo: una teoría de aprendizaje para la era digital. 10. https://ateneu.xtec.cat/wikiform/wikiexport/_media/cursos/tic/s1x1/modul_3/c onectivismo.pdf 